\title{
Augmenting Self-Stability: Height Control of a Bernoulli Ball via Bang-Bang Control
}

\author{
Toby Howison ${ }^{1}$, Fabio Giardina ${ }^{2}$ and Fumiya Iida ${ }^{1}$
}

\begin{abstract}
Mechanical self-stability is often useful for controlling systems in uncertain and unstructured environments because it can regulate processes without explicit state observation or feedback computation. However, the performance of such systems is often not optimised, which begs the question how their dynamics can be naturally augmented by a control law to improve performance metrics. We propose a minimalistic approach to controlling mechanically self-stabilising systems by utilising model-based, feedforward bang-bang control at a global level and self-stabilizing dynamics at a local level. We demonstrate the approach in the height control problem of a sphere hovering in a vertical air jet-the so-called Bernoulli Ball. After developing a model to study the system and theoretically proving global asymptotic stability, we present the augmented controller and show how to enhance performance measures and plan behaviour. Our physical experiments show that the proposed control approach has a reduced time-to-target compared to the uncontrolled system without loss of stability (ranging from a 2.4 to 4.4 fold improvement) and that we can plan sequences of target positions at will.
\end{abstract}

\section{INTRODUCTION}

There is a long-standing interest in utilizing mechanical self-stability-henceforth termed self-stability-for the purpose of dynamic motion control [1], [2]. Many animals are known to take advantage of their mechanical dynamics to maintain stability during legged locomotion, especially in unstructured and uncertain environments [3]. Inspired by this, there have been a range of studies on designing robust robotic systems, e.g. in legged locomotion [4] where self-stabilising passive dynamics are exploited [5]-[8], or the "Blind Juggler" [9], [10], which exploits the passive dynamics of a ball bouncing on a parabolic surface to juggle multiple balls without any feedback. Self-stabilizing properties can even be observed in a bicycle [11].

In general, exploiting self-stability benefits motion control in three ways. First, self-stability offers motion regulation mechanisms "for free", without the need for sensing and computation for control [1], [8]. Second, self-stability can be exploited for energy efficiency, even in actively controlled applications. For example, exploiting pendulum-like [8], [12] or elastic [9], [12], [13] passive dynamics in legged locomotion can yield efficiencies similar to biological systems. Third, because of the simplicity of self-stability, we can increase the complexity of system behaviours without adding extra mechanisms for sensory-motor coordination.

*This work was supported by The Mathworks and the EPSRC Grant RG92738

${ }^{1}$ Department of Engineering, University of Cambridge, Cambridge UK. [th533, fi224] @cam.ac.uk

${ }^{2}$ School of Engineering and Applied Sciences, Harvard University, Cambridge USA. giardina@seas . harvard.edu
Despite these advantages, self-stabilizing systems are generally considered challenging when designing active control mechanisms, because the system behaviours are dictated by their intrinsic mechanical dynamics, which we cannot directly change [14]. Additionally, actuation and control signal latency may be too large to control the fast dynamics via feedback control. Various methods exist for the active control of self-stabilising systems. These include energy shaping methods [15] or LQR-Trees [16]. While effective, these approaches require precise system models and high control bandwidth, which can pose an insurmountable challenge in noisy real-world systems.

In this paper, we propose a minimalistic control approach which aims to augment the performance of self-stable mechanical systems without loss of their intrinsic stabilizing properties. We demonstrate the process of control implementation in the case of the height control problem of a sphere hovering in a vertical airflow-the so-called Bernoulli Ball. Though this is an interesting control challenge, it has not been studied intensively in the past; the authors discovered one other study [17]. We develop a dynamics model and show that the self-stabilizing trajectories of the real system can be augmented by implementing a model-based bangbang controller that improves time-to-target considerably without a loss of stability. The advantage of this approach is in its robustness to disturbances, simplicity of application, and its capacity to plan fast switches between target positions. We argue that this approach generalizes to the control problem of many real-world systems with self-stabilising properties.

The paper is organised as follows. First, we introduce the model of the Bernoulli Ball, which is followed by a Lyapunov stability analysis to verify self-stability. A bangbang control method is then derived and tested in simulation. Finally, experimental results of the model-based controller are presented along with comparative analysis of the proposed model.

\section{METHODS}

Before thinking about controlling a system with any means of actuation, it is advisable to maximize its mechanical self-stability. There are, unfortunately, no design principles for self-stability as its cause is not easily understood even for seemingly simple systems such as bicycles [11]. In the case of the Bernoulli ball, the self-stabilizing properties of the system can significantly simplify the mathematical description for the purpose of control, despite the complex three dimensional body-fluid interaction. In the following 
sections we will outline how to model, analyze, and synthesise a controller for a self-stable system exemplified by the Bernoulli ball.

\section{A. Dynamics Modelling}

The Bernoulli-Ball system-as shown in Figure 1aexhibits two forms of self-stability. Firstly, it is self-stable in the horizontal plane; when perturbed horizontally the ball returns to it's original position. This is commonly explained using Bernoulli's principle, which states that as the speed of a fluid increases, the fluid pressure decreases. Hence, the pressure within an airflow is lower than the surrounding environment, creating a self-stabilising force about the jet centerline [18]. Secondly, it is self-stable in the vertical direction; when released into the airflow, the ball will eventually settle around a nominal height. This is due to the force balance between drag and the ball mass, and the dissipative effect of moving in the airflow. To simplify matters, we first assume that motion in the horizontal plane $x-y$ and the vertical direction $z$ is decoupled. We make this assumption because the horizontal self-stability acts as a feedback loop, keeping the ball centred in the flow.

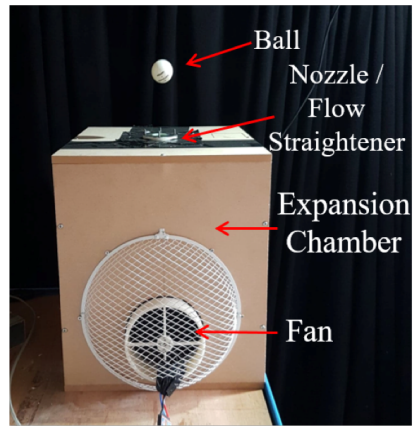

(a)

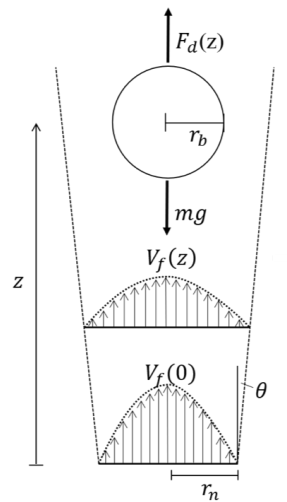

(b)
Fig. 1: (a) Bernoulli-Ball system. Air is forced into an expansion chamber and through a flow straightening nozzle. A ping-pong ball hovers on the vertical air jet. (b) Schematic of dynamics modelling assumptions.

The vertical dynamics are the result of complex interactions between the ball, airflow and environment, meaning the governing equations are non-linear. Here, we present a simplified model of the system dynamics. We start by considering the force balance on the ball in the vertical direction,

$$
m \ddot{z}=F_{d}(z, \dot{z})+F_{b}-m g
$$

where $z$ is the vertical distance between the nozzle outlet and ball centre of mass, $F_{d}(z, \dot{z})$ is the drag force on the ball at a height $z$ and velocity $\dot{z}, F_{b}$ is the buoyant force due to the weight of displaced air and $m g$ is the ball weight. The buoyant force is minimal, so we have $F_{b} \approx 0$.

We assume that the air-jet flow expands conically from the nozzle with a cone angle $\theta$ and that the velocity profile $V_{f}(z)$ is approximately parabolic in the radial direction, as shown in Figure $1 \mathrm{~b}$. We define $\bar{V}_{f}(z)$ as the mean flow velocity at a height $z$, with the mean velocity at the nozzle outlet being denoted by $V_{0}=\bar{V}_{f}(0)$. Applying flow continuity over $z$ yields an expression for $\bar{V}_{f}(z)$,

$$
\bar{V}_{f}(z)=\frac{V_{0} r_{n}^{2}}{\left(r_{n}+z \tan \theta\right)^{2}}
$$

where $r_{n}$ is the nozzle radius.

The mean nozzle outlet velocity changes in response to a change in fan power, which is controlled using a PWM signal denoted by $u$. The dynamics of the fan, expansion chamber and airflow, and delays in the control PC and microcontroller introduce latency into the system, which manifests as a delay between a change in control input and a change in the ball force balance. Modelling each of these transient components is challenging, so we model all transience as a delay in $u$ reaching a demanded value, defined as $u^{*}$. We set a first-order constraint on the time derivative of $u$, namely

$$
\frac{d u}{d t}=k_{t}\left(u^{*}-u\right)
$$

where $k_{t}$ is a time constant to be determined.

We define $f(u)$ as the yet-to-be-determined mapping between $u$ and $V_{0}$. Therefore,

$$
\bar{V}_{f}(z)=\frac{f(u) r_{n}^{2}}{\left(r_{n}+z \tan \theta\right)^{2}}
$$

Therefore, we can reformulate (1) as

$$
m \ddot{z}=k_{S} \frac{1}{2} C_{d} \pi r_{b}^{2} \rho\left(\frac{f(u) r_{n}^{2}}{\left(r_{n}+z \tan \theta\right)^{2}}-\dot{z}\right)^{2}-m g
$$

where $k_{s}=\operatorname{sgn}\left(\bar{V}_{f}(z)-\dot{z}\right)$. We recast (5) in its state-space representation. There are three states

$$
\mathbf{x}=\left[\begin{array}{c}
z \\
\dot{z} \\
u
\end{array}\right]=\left[\begin{array}{l}
x_{1} \\
x_{2} \\
x_{3}
\end{array}\right]
$$

Therefore, the state-space representation of the system is

$$
\frac{d \mathbf{x}}{d t}=\left[\begin{array}{c}
x_{2} \\
k_{s} \frac{1}{2} C_{d} \pi r_{b}^{2} \rho\left(\frac{f\left(x_{3}\right) r_{n}^{2}}{\left(r_{n}+x_{1} \tan \theta\right)^{2}}-x_{2}\right)^{2}-m g \\
k_{t}\left(u^{*}-x_{3}\right)
\end{array}\right]
$$

where $r_{b}, \rho, r_{n}, m$ and $g$ are known or easily measurable, and $C_{d}, f(\mu), \theta$ and $k_{t}$ are unknown parameters. Equation (7) can be easily integrated using the MATLAB ordinary differential equation solver ODE 45.

\section{B. System Identification}

The unknown parameters were identified by measuring the hovering height and flow velocities for a range of control inputs. Each parameter was tuned to best best match the modelled and experimentally determined values. Table I shows all system parameters. 


\begin{tabular}{|c|c|}
\hline Parameter & Value \\
\hline$r_{b}$ & $20 \mathrm{~mm}$ \\
$r_{n}$ & $15 \mathrm{~mm}$ \\
$\rho$ & $1.22 \mathrm{kgm}^{-3}$ \\
$m$ & $2.7 \mathrm{~g}^{-2}$ \\
$g$ & $9.81 \mathrm{~ms}^{-2}$ \\
$\theta$ & $1^{o}$ \\
$C_{d}$ & 0.185 \\
$k_{t}$ & $3 s^{-1}$ \\
$f(u)$ & $-30.24 u^{2}+47.91 u+2.45$ \\
\hline
\end{tabular}

TABLE I: Unknown system parameters.

\section{Global Asymptotic Stability}

We have stated that the system exhibits self-stabilizing properties in the vertical direction. Here, we present an analysis that proves this self-stability analytically in the uncontrolled case with smooth right hand side of (7). Specifically, we show that for any constant and converged control input $u=u^{*}$, the system will always converge to an equilibrium state $\mathbf{x}^{*}=\left[\begin{array}{lll}z^{*} & 0 & u^{*}\end{array}\right]^{T}$, providing the initial conditions $\mathbf{x}_{\text {initial }}$ lie on the domain $D_{\mathbf{x}}=\left\{\mathbf{x} \mid x_{1}>-r_{n} / \tan \theta\right\}$; a property known as global asymptotic stability. Note that $D_{\mathbf{x}}$ is a positive invariant set with respect to the dynamics (7) for positive fan speeds due to the singularity at $x_{1}=-r_{n} / \tan \theta$. Of course, in reality $x_{1} \geq 0$ as the ball cannot travel below the nozzle, so any physically plausible state $\mathrm{x}$ will by definition lie in $D_{\mathbf{x}}$.

First, we define $\mathbf{y}$ as the state variable measured around $\mathbf{x}^{*}$, i.e.

$$
\mathbf{y}=\left[\begin{array}{c}
z-z^{*} \\
\dot{z}
\end{array}\right]=\left[\begin{array}{l}
y_{1} \\
y_{2}
\end{array}\right]
$$

Since we assume the air flow speed has converged to a constant positive value, we neglect the third system state and assume $u=u^{*}$. Reformulating (7) in this form yields

$$
\dot{\mathbf{y}}=\left[\begin{array}{c}
y_{2} \\
k_{s} \frac{C_{d} \rho r_{b}^{2} \pi}{2 m}\left(\frac{f\left(u^{*}\right) r_{n}^{2}}{\left(r_{n}+\left(y_{1}+z^{*}\right) \tan (\theta)\right)^{2}}-y_{2}\right)^{2}-g
\end{array}\right]
$$

valid on the domain $D_{\mathbf{y}}=\left\{\mathbf{y} \mid y_{1}>-r_{n} / \tan \theta-z^{*}\right\}$. For brevity, we denote (9) as

$$
\dot{\mathbf{y}}=\left[\begin{array}{c}
y_{2} \\
F\left(y_{1}, y_{2}\right)
\end{array}\right]
$$

We prove the equilibrium $\mathbf{y}^{*}=\left[\begin{array}{ll}0 & 0\end{array}\right]^{T}$ is globally asymptotically stable by employing the Lyapunov stability criterion, which requires that a Lyapunov candidate function $L(\mathbf{y})$ exists such that

- $L(\mathbf{0})=0$

- $L(\mathbf{y})>0 \forall \mathbf{y} \in D_{\mathbf{y}} \backslash\{\mathbf{0}\}$

- $\dot{L}(\mathbf{y})<0 \forall \mathbf{y} \in D_{\mathbf{y}} \backslash\{\mathbf{0}\}$, i.e. $L$ decreases along trajectories.

We construct the Lyapunov function

$$
L\left(y_{1}, y_{2}\right)=\frac{1}{2} m y_{2}^{2}+W\left(y_{1}\right)
$$

where $d W / d y_{1}=-m F\left(y_{1}, 0\right)$. Hence, $L\left(y_{1}, y_{2}\right)$ is the sum of kinetic and potential energy, deriving the latter from the sum of gravitational and drag forces but neglecting the effect of the ball velocity $y_{2}$.

To find $W$, we integrate $-m F\left(y_{1}, 0\right)$, defining the constant of integration such that $W(0)=0$, to give

$$
W\left(y_{1}\right)=-m\left(\frac{K_{1}}{\tan \theta\left(K_{2}+y_{1} \tan \theta\right)^{3}}+\frac{K_{1}}{\tan \theta K_{2}^{3}}-g y_{1}\right)
$$

where $K_{1}=C_{d} \rho r_{b}^{2} \pi f^{2}\left(u^{*}\right) r_{n}^{4} / 6 m$ and $K_{2}=r_{n}+z^{*} \tan \theta$. Hence, $L(\mathbf{0})=0$.

First, we show the state $\mathbf{y}=\mathbf{0}$ is the unique minimum for $L \in D_{\mathbf{y}}$. When in equilibrium, $F(0,0)=0$. Since $d W / d y_{1}=-m F\left(y_{1}, 0\right), W$ is stationary at this point. Moreover, for $y_{1}<0, d W / d y_{1}<0$ and for $y_{1}>0$, $d W / d y_{1}>0$. Hence, the origin is the unique minimum. Similarly, the origin is a global minimum for the term $\frac{1}{2} m y_{2}^{2}$. Hence, $L$ is the unique minimum for $L \in D_{\mathbf{y}}$.

The time derivative of the Lyapunov function is

$$
\dot{L}=m y_{2} \dot{y}_{2}+\frac{d W}{d y_{1}} \dot{y_{1}}
$$

which, after substituting for $y_{1}, d W / d y_{1}$ and $\dot{y}_{2}$ yields

$$
\begin{aligned}
\dot{L} & =m y_{2}\left(F\left(y_{1}, y_{2}\right)-F\left(y_{1}, 0\right)\right) \\
& =y_{2} K_{3}\left(\operatorname{sgn}\left(\bar{V}_{f}-y_{2}\right)\left(\bar{V}_{f}-y_{2}\right)^{2}-\bar{V}_{f}^{2}\right)
\end{aligned}
$$

where $K_{3}=\frac{C_{d} \rho r_{b}^{2} \pi}{2 m}$ and $\bar{V}_{f}=\left(f\left(u^{*}\right) r_{n}^{2}\right) /\left(\left(r_{n}+\left(y_{1}+\right.\right.\right.$ $\left.\left.\left.z^{*}\right) \tan (\theta)\right)^{2}\right)$.

Now, we show that $L$ decreases along trajectories, and in fact $\dot{L}<0$ except instantaneously when $y_{2}=0$. We prove this by considering four cases dictated by the signum function in (14).

Case 1: $0<y_{2} \leq \bar{V}_{f} \rightarrow \operatorname{sgn}\left(\bar{V}_{f}-y_{2}\right)=1$ Since $\bar{V}_{f}^{2}>$ $\left(\bar{V}_{f}-y_{2}\right)^{2}$, the right hand term in (14) dominates so $\dot{L}<0$.

Case 2: $0<\bar{V}_{f}<y_{2} \rightarrow \operatorname{sgn}\left(\bar{V}_{f}-y_{2}\right)=-1$ Both terms in (14) are negative so $\dot{L}<0$.

Case 3: $y_{2}<0 \rightarrow \operatorname{sgn}\left(\bar{V}_{f}-y_{2}\right)=1$ The left term is positive and dominates in (14), but $y_{2}$ is negative so $\dot{L}<0$.

Case 4: $y_{2}=0 \rightarrow \dot{L}=0$. This violates the Lyapunov criterion as clearly $\dot{L} \leq 0$, i.e. not strictly negative definite; however, we know from (10) that when $y_{2}=0, \dot{y}_{2}=$ $F\left(y_{1}, 0\right) \neq 0$. Hence, $\dot{L}$ is only zero instantaneously and the dynamics always return to the provably stable regions shown in cases 1-3. (In fact, this point indicates an inflection point for $L$ ). These conditions still qualify for LaSalle's theorem, which is a proof for global asymptotic stability of the equilibrium $\mathbf{y}^{*}$ in a positive invariant set $D_{\mathbf{y}}$.

Hence, all conditions have been met (with the modification of $\dot{L}$ requiring to be only negative semidefinite on $D_{\mathbf{y}}$ to qualify for LaSalle's theorem) and the equilibrium $\mathbf{y}^{*}$ is globally asymptotically stable on $D_{\mathbf{y}}$ which is equivalent to $\mathrm{x}^{*}$ being globally asymptotically stable on $D_{\mathbf{x}}$ for converged fan speeds.

\section{Bang-Bang Control Algorithm}

While state feedback commonly helps stabilizing the system in theory, the large latencies in actuation and sensory signals and considerable velocity fluctuation due to fluid 


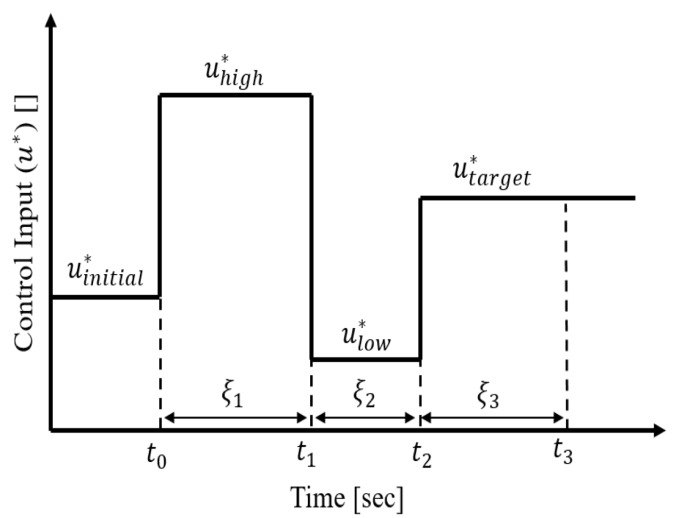

Fig. 2: Example of a bang-bang control policy.

turbulence render state feedback hard to deal with in the Bernoulli-Ball system, unless considerable investments into sensory equipment, actuation, and controller synthesis are made. Therefore, the control approach for the Bernoulli ball as presented here is to use a model-based feedforward bang bang approach inspired from time-optimal control in linear systems. Not only do we need less equipment for control (no sensors or fast actuators), but we find a highly effective and robust control law to minimize time-to-target of the Bernoulli ball. Figure 2 shows an example of a bang-bang control policy.

It is advantageous to augment the self-stabilizing dynamics of the Bernoulli-Ball using a bang bang controller for a number of reasons. First, self-stabilizing systems reliably follow certain trajectories under certain control inputs. Bangbang control harnesses these trajectories, which by nature are reliable, hence making the control policy robust to disturbances. Second, the control bandwidth requirement is minimal - there is no requirement for high-frequency sensing and computation of control inputs-meaning control policies can be calculated a priori. Third,the control is defined with few discrete parameters that give rise to a certain trajectory. These types of control problems are efficiently optimized using numerical solvers.

We seek to move the system from an initial state $\mathbf{x}_{\text {initial }}$ to a target state $\mathbf{x}_{\text {target }}$. In our system, these states correspond to target hovering heights; for a target height $z_{\text {target }}$ which corresponds to a control input of $u_{\text {target }}^{*}, \mathbf{x}_{\text {target }}=\left[\begin{array}{lll}z_{\text {target }} & 0 & u_{\text {target }}^{*}\end{array}\right]^{T}$. It is important to note here that because of the transient system dynamics, control demands are included in the target state.

We implement a numerical switching time computation method, similar to the method outlined in [19]. The proposed bang-bang controller switches between the maximal, minimal and target control inputs $u_{\text {high }}^{*}, u_{\text {low }}^{*}$ and $u_{\text {target }}^{*}$ at a set of three predetermined switching times, as demonstrated in Figure 2a. We denote the $i$ th switching time as $t_{i}$ and the initial and final times as $t_{0}$ and $t_{f}$, such that $0=t_{0} \leq t_{1} \leq t_{2} \leq t_{3}=t_{f}$. The system trajectory between each switching time, denoted by $\mathbf{x}_{i}(t)$, is called the ith bang arc, so the total trajectory $\mathbf{x}(t)$ for $0 \leq t \leq t_{f}$ is the concatenation of $\mathbf{x}_{i}(t)$ and ends

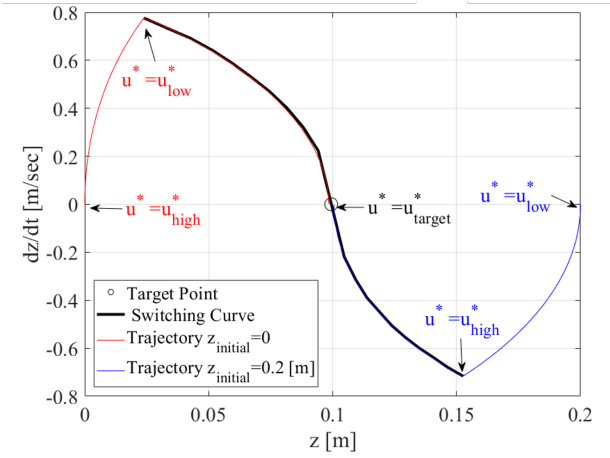

(a) Switching curve for idealised system with no latency.

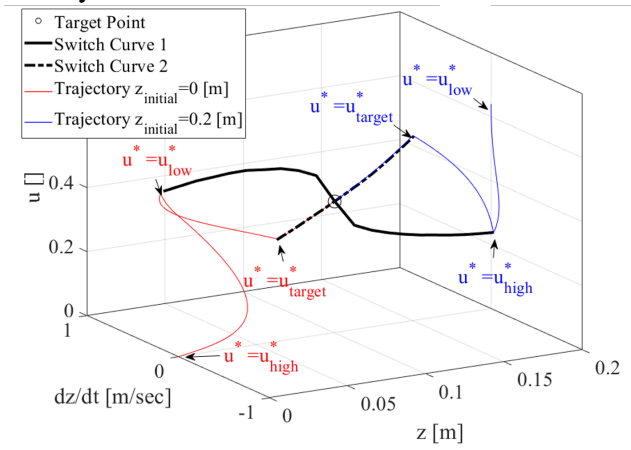

(b) Three-dimensional switching curve for realworld system with latency.

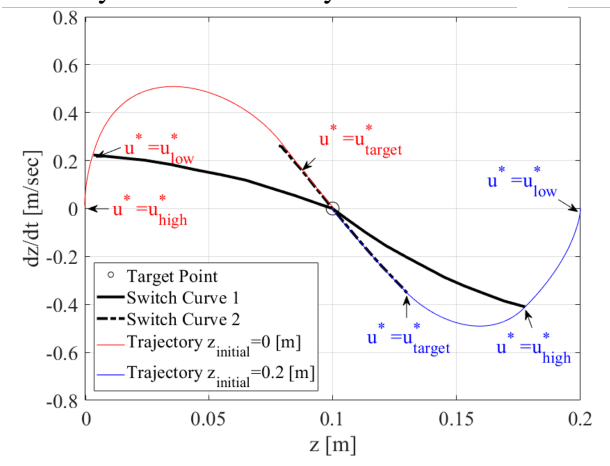

(c) Two-dimensional switching curve projection for real-world system with latency.

Fig. 3: Idealised and real-world bang-bang control switching curves for $z_{\text {target }}=0.1 \mathrm{~m}$, showing trajectories for $z_{\text {initial }}=0$ $\mathrm{m}$ and $z_{\text {initial }}=0.2 \mathrm{~m}$

at the final state $\mathbf{x}_{\mathrm{f}}$. The arc time $\xi_{i}$ is the time spent on each bang arc, which together are stored in the arc time vector $\boldsymbol{\xi}=\left[\begin{array}{lll}\xi_{1} & \xi_{2} & \xi_{3}\end{array}\right]^{T}$. Arc times $\xi_{1}$ and $\xi_{2}$ represent the time spent at $u_{\text {high }}^{*}$ and $u_{\text {low }}^{*}$, or vice versa, while $\xi_{3}$ is a predefined time used to set a consistent point to measure control performance.

We seek to determine $\boldsymbol{\xi}$ such that the error between $\mathbf{x}_{\mathrm{f}}$ and $\mathbf{x}_{\text {target }}$ is minimized. We formally define this using a control cost function $g(\boldsymbol{\xi})$, representing the absolute distance between $\mathbf{x}_{\mathrm{f}}$ and $\mathbf{x}_{\text {target}}$,

$$
g(\boldsymbol{\xi})=\left\|\mathbf{x}_{f}-\mathbf{x}_{\text {target }}\right\|
$$


Hence, we seek to solve the minimization problem

$$
\min _{\boldsymbol{\xi}}=g(\boldsymbol{\xi})
$$

Due to the non-linearity of $g(\boldsymbol{\xi})$ an analytical solution to (16) is not necessarily tractable, so we employ the MATLAB fminsearch numerical optimisation function.

For any given $z_{\text {target }}$ we can numerically construct a set of switching curves that indicate where in the state-space the controller should switch between the three control inputs. Figure 3a-c shows these switching curve for $z_{\text {target }}=0.1$ $\mathrm{m}$, with example trajectories for $z_{\text {initial }}=0 \mathrm{~m}$ and $z_{\text {initial }}=$ $0.2 \mathrm{~m}$. Figure $3 \mathrm{a}$ shows the switching curve for an idealised system with no latency, while Figure $3 b-c$ shows the $3 \mathrm{D}$ and projected 2D switching curves for the real world system.

\section{EXPERIMENTAL SET-UP}

A high-power brushless motor (Diatone M2205 2300KV) fitted with a 3-blade propeller (Diatone 5045) and a constant input voltage of $15 \mathrm{VDC}$ was used to propel air into an expansion chamber then through a circular nozzle positioned on top of the chamber. To stabilize the outlet jet, a flow straightener was fitted under the nozzle. Control was achieved using an electronic speed control (HobbyKing 20A ESC 3A UBEC) connected to an Arduino UNO interfaced with MATLAB. A Pulse-width modulation (PWM) signal was used to vary motor speed by changing the available current. The ball height was measured using a high frame rate webcam (Logitech BRIO) capable of delivering 90120 frames per second. In each frame the ball was located using a simple thresholding algorithm, after which the height was determined using the predetermined camera parameters. Figure 1a shows the setup.

\section{EXPERIMENTAL RESULTS}

The controller was used to calculate the switching times $z_{\text {initial }}=0 \mathrm{~m}$ and $z_{\text {target }}=0.1,0.15,0.2 \mathrm{~m}$. The calculated policies were applied in the real system, as were the corresponding open-loop control policies for the same target heights. Figure 4 shows snapshot images of these controlled responses, while Figure 5 shows the state space controlled and open-loop responses for $z_{\text {target }}=0.1,0.15$ and $0.2 \mathrm{~m}$. In all cases, the open-loop response is characterized by a spiralling trajectory ending in steady-state behaviour, which manifests as an oscillation around the target height. Conversely, the controlled response is characterized by a curved trajectory directly to steady-state.

The time taken for the system to reach its steady state behaviour - the settling time, $t_{s}$-in the open-loop and controlled response was measured. The factor of improvement in $t_{s}$ between the controlled and open loop response was 2.4, 3.6 and 4.4 for $z_{\text {target }}=0.1,0.15,0.2 \mathrm{~m}$ respectively. Table II shows these results.

This difference in improvement based on $z_{\text {target }}$ can be attributed to a number of factors. As discussed previously, the dynamics model is less accurate at duty-cycles, meaning the bang-bang control policy is likely to be less effective when $z_{\text {target }}$ is lower. Furthermore, because the system dynamics are highly non-linear we expect the relationship between $z_{\text {target }}$ and $t_{s}$ to also exhibit non-linearity.

\begin{tabular}{|c|c|c|c|}
\hline$z_{\text {target }} \mathrm{m}$ & Open-Loop $t_{s}[\mathrm{~s}]$ & Controlled $t_{s}[\mathrm{~s}]$ & Improv. Factor \\
\hline 0.1 & 2.3 & 0.98 & 2.4 \\
0.15 & 4.1 & 1.12 & 3.6 \\
0.2 & 5.2 & 1.20 & 4.4 \\
\hline
\end{tabular}

TABLE II: Open-loop and controlled settling time and improvement factor, for $z_{\text {target }}=0.1,0.15$ and $0.2 \mathrm{~m}$ and $z_{\text {initial }}=0 \mathrm{~m}$.

The control policy was also tested for consecutive trajectory following, where $z_{\text {target }}$ switched every 5 seconds and applied open-loop control for comparison, as shown in Figure 6 and Supplementary Video 1. This is a more challenging task, as the initial state is subject to high amounts of uncertainty. Qualitatively, we see that the controlled response significantly reduces $t_{s}$ for each height change.

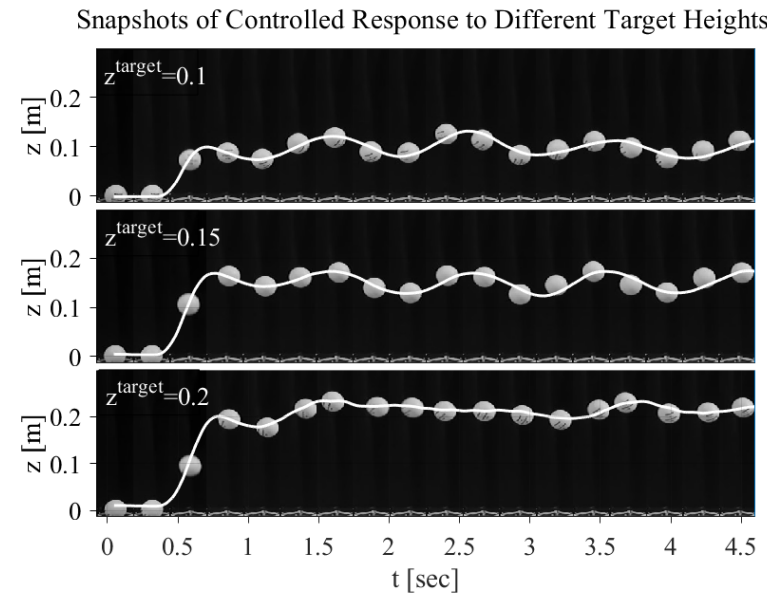

Fig. 4: Snapshot images recorded over five seconds of controlled response for $z_{\text {target }}=0.1,0.15$ and $0.2 \mathrm{~m}$ and $z_{\text {initial }}=0 \mathrm{~m}$, corresponding to Figure $5 \mathrm{~d}, \mathrm{e}, \mathrm{f}$.

\section{Discussion \& Conclusion}

We have shown that augmenting the self-stability of the Bernoulli-Ball via Bang-Bang control can significant improve the time to target of the system when changing heights. This example demonstrates how fast dynamics can be controlled in a robotic system without the need for highbandwidth control. We have to be careful in generalizing the results to other robotic systems as this approach can technically only guarantee stability in the presence of a globally asymptotically stable equilibrium in the uncontrolled system. However, in the real world experiment we have achieved stable performance in spite of the ground restriction to positive ball heights, which shows that in practice the approach can work for locally self-stable equilibria, allowing its applicability to a larger class of problems including passive dynamic walking [5] and bicycle stability [11]. We have studied exclusively the vertical dimension of the system. The horizontal component requires different modelling 


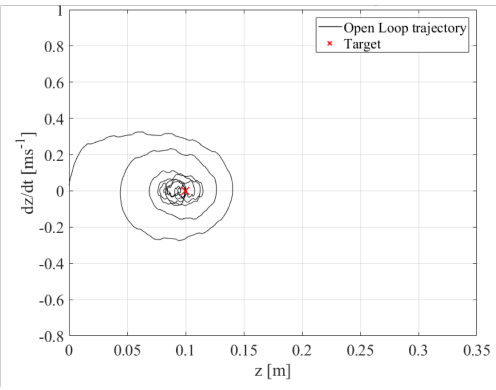

(a) $\mathrm{z}_{\text {target }}=0.1 \mathrm{~m}$.

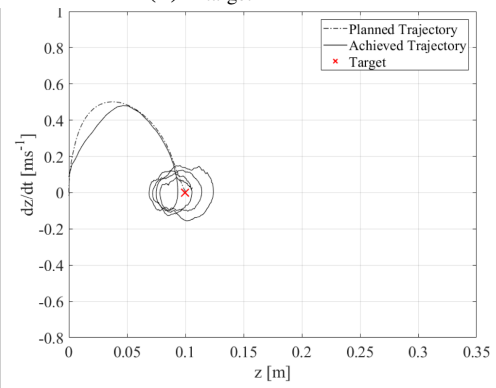

(d) $\mathrm{z}_{\text {target }}=0.1 \mathrm{~m}$.

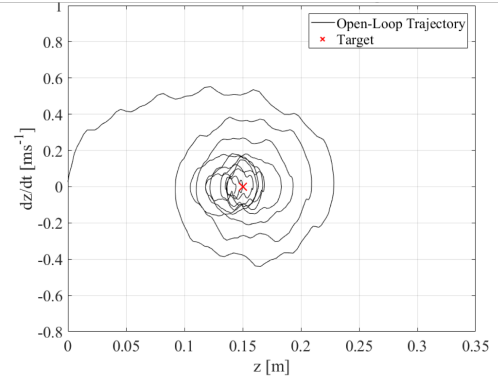

(b) $\mathrm{z}_{\text {target }}=0.15 \mathrm{~m}$.

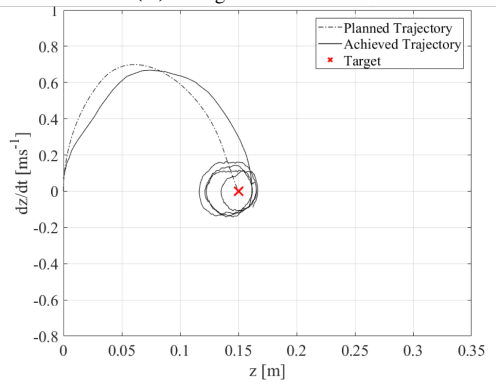

(e) $\mathrm{z}_{\text {target }}=0.15 \mathrm{~m}$.

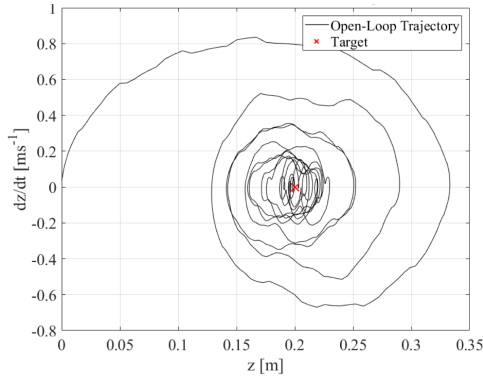

(c) $\mathrm{z}_{\text {target }}=0.2 \mathrm{~m}$.

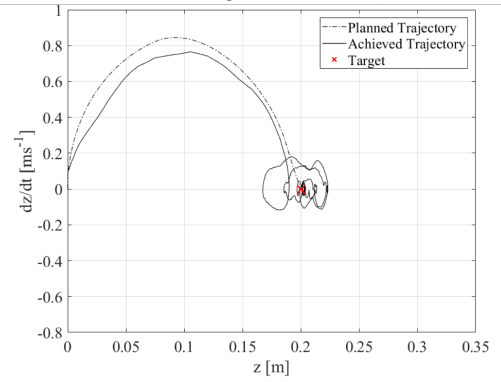

(f) $\mathrm{z}_{\text {target }}=0.2 \mathrm{~m}$.

Fig. 5: Open-loop $(\mathrm{a}, \mathrm{b}, \mathrm{c})$ and controlled $(\mathrm{d}, \mathrm{e}, \mathrm{f})$ response for $\mathrm{z}_{\text {target }}=0.1,0.15$ and $0.2 \mathrm{~m}$
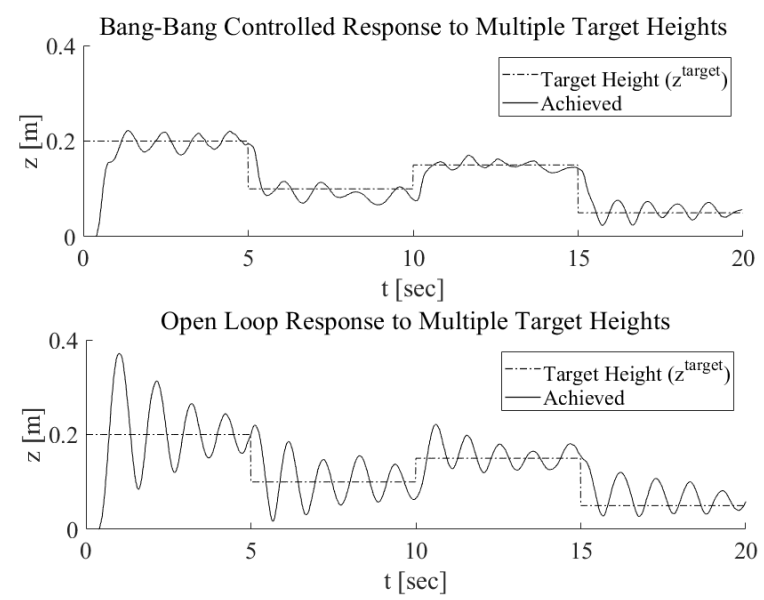

Fig. 6: Controlled and open-loop response to consecutive trajectory following.

approaches and more actuators for control which we will study in future work.

Using bang-bang control in the Bernoulli-Ball system is a simple yet effective approach to improve the performance, and is an approach that could generalises to many selfstable robotic systems. It outsources the difficult tasks of stabilizing control to the mechanical feedback loop, leaving the trajectory planning via bang-bang control to a numerical optimisation which can be solved off-line. The high performance and robustness of the presented results thus suggests that the focus on the mechanical design to allow for selfstability can be key to overcoming the difficulties arising in control of systems with fast dynamics.

In conclusion, we believe any robotic system should be designed to maximize self-stabilizing effects as they can help improve robustness and reduce actuator efforts. Based on the example of the Bernoulli ball, we anticipate this work to provide a modelling and control guide for real world systems with actuation and signal latency, fast dynamics, and noise. Furthermore, we present the first model-based approach to control a Bernoulli ball via bang-bang control and we prove stability of the self-stable system in the sense of Lyapunov.

\section{REFERENCES}

[1] R. Pfeifer, M. Lungarella, and F. Iida, "Self-organization, embodiment, and biologically inspired robotics," science, vol. 318, no. 5853, pp. 1088-1093, 2007.

[2] R. Blickhan, A. Seyfarth, H. Geyer, S. Grimmer, H. Wagner, and M. Günther, "Intelligence by mechanics," Philosophical Transactions of the Royal Society A: Mathematical, Physical and Engineering Sciences, vol. 365, no. 1850, pp. 199-220, Jan. 2007. [Online]. Available: https://doi.org/10.1098/rsta.2006.1911

[3] A. J. Ijspeert, "Biorobotics: Using robots to emulate and investigate agile locomotion," science, vol. 346, no. 6206, pp. 196-203, 2014.

[4] F. Iida and A. J. Ijspeert, "Biologically inspired robotics," in Springer Handbook of Robotics. Springer, 2016, pp. 2015-2034.

[5] T. McGeer et al., "Passive dynamic walking," I. J. Robotic Res., vol. 9, no. 2, pp. 62-82, 1990.

[6] M. W. Spong, J. K. Holm, and D. Lee, "Passivity-based control of bipedal locomotion," IEEE Robotics \& Automation Magazine, vol. 14, no. 2, pp. 30-40, 2007.

[7] A. Goswami, B. Espiau, and A. Keramane, "Limit cycles in a passive compass gait biped and passivity-mimicking control laws," Autonomous Robots, vol. 4, no. 3, pp. 273-286, 1997.

[8] S. Collins, A. Ruina, R. Tedrake, and M. Wisse, "Efficient bipedal robots based on passive-dynamic walkers," Science, vol. 307, no. 5712, pp. 1082-1085, 2005.

[9] P. Reist and R. D'Andrea, "Design of the pendulum juggler." in ICRA, 2011, pp. 5154-5159.

[10] — - "Design and analysis of a blind juggling robot," IEEE Transactions on Robotics, vol. 28, no. 6, pp. 1228-1243, 2012.

[11] J. Kooijman, J. P. Meijaard, J. M. Papadopoulos, A. Ruina, and A. Schwab, "A bicycle can be self-stable without gyroscopic or caster effects," Science, vol. 332, no. 6027, pp. 339-342, 2011. 
[12] F. Iida and R. Tedrake, "Minimalistic control of biped walking in rough terrain," Autonomous Robots, vol. 28, no. 3, pp. 355-368, 2010.

[13] S. G. Nurzaman, X. Yu, Y. Kim, and F. Iida, "Guided self-organization in a dynamic embodied system based on attractor selection mechanism," Entropy, vol. 16, no. 5, pp. 2592-2610, 2014.

[14] R. R. Burridge, A. A. Rizzi, and D. E. Koditschek, "Sequential composition of dynamically dexterous robot behaviors," The International Journal of Robotics Research, vol. 18, no. 6, pp. 534-555, 1999.

[15] M. W. Spong, "Passivity based control of the compass gait biped," IFAC Proceedings Volumes, vol. 32, no. 2, pp. 506-510, 1999.

[16] R. Tedrake, I. R. Manchester, M. Tobenkin, and J. W. Roberts, "Lqrtrees: Feedback motion planning via sums-of-squares verification," The International Journal of Robotics Research, vol. 29, no. 8, pp. 1038$1052,2010$.

[17] S. S. Nudehi, S. Dehmlow, and D. Clark, "Position control of a floating ball in a vertical air stream," in ASME 2017 International Mechanical Engineering Congress and Exposition. American Society of Mechanical Engineers, 2017, pp. V04AT05A002-V04AT05A002.

[18] P. M. Gerhart, A. L. Gerhart, and J. I. Hochstein, Munson, Young and Okiishi's Fundamentals of Fluid Mechanics, Binder Ready Version. John Wiley \& Sons, 2016.

[19] C. Y. Kaya and J. L. Noakes, "Computations and time-optimal controls," Optimal control applications and methods, vol. 17, no. 3, pp. 171-185, 1996. 